\title{
Leishmaniose visceral canina: caso alóctone no município de Resende, estado do Rio de Janeiro, Brasil*
}

\section{Canine visceral leishmaniasis: alochthonous case in Resende municipality, state of Rio de Janeiro, Brazil}

\author{
Tassia Cristina Bello de Vasconcelos, ${ }^{* *}$ Flávio Jacob Alves, ${ }^{* * *}$ Arthur Augusto Velho Mendes Junior, ${ }^{* * * *}$ \\ Maria de Fátima Madeira, ${ }^{* * * * *}$ Fabiano Borges Figueiredo****
}

\begin{abstract}
Resumo
A leishmaniose visceral (LV) é uma endemia em franca expansão geográfica. Este relato apresenta um caso de importação de LV canina para o município de Resende, estado do Rio de Janeiro, onde não havia até o momento registro de casos humanos ou caninos da parasitose. O animal era oriundo do estado de Minas Gerais, área endêmica de LV, e apresentou clínica compatível com o processo patológico em questão. O diagnóstico foi realizado por meio de avaliação sorológica com o teste rápido imunocromatográfico DPP ${ }^{\circledR}$ (Dual Path Platform) e teste de ELISA, ambos com resultado positivo, além de cultura parasitológica em Meio NNN acrescido de meio Schneider a partir de punções de medula óssea e linfonodos, biópsias de pele íntegra, lesões cutâneas, linfonodo e baço, obtendo-se o isolamento de formas promastigotas compatíveis com Leishmania spp., com posterior caracterização de Leishmania (Leishmania) chagasi através de eletroforese de isoenzimas. O levantamento entomológico com utilização de armadilhas luminosas do tipo CDC, realizado no peridomicílio três vezes por semana durante três meses, não evidenciou a presença de flebotomíneos, e o inquérito sorológico canino, procedido em 144 animais ao redor da residência do caso positivo de LV, descartou novos casos da parasitose por meio do teste rápido imunocromatográfico $\mathrm{DPP}^{\circledR}$ e/ou confirmação por ELISA. Os resultados e o histórico de deslocamento do animal caracterizam o caso como alóctone, expõem a fragilidade em relação ao controle de trânsito de animais e ressaltam a importância das ações de Vigilância para a parasitose em áreas indenes.
\end{abstract}

Palavras-chave : diagnóstico, cão, Leishmania.

\begin{abstract}
The visceral leishmaniasis (VL) is an endemic parasitosis in intense geographic spread. This report presents a case of canine $\mathrm{VL}$ importation for the city of Resende, state of Rio de Janeiro, where had not been reported cases of human or canine disease yet. The animal was from Minas Gerais state, endemic area for VL, and showed compatible clinical signs of this disease. The diagnosis was made by serology with DPP $®$ (DUAL PATH PLATFORM) immunochromatographic rapid test and ELISA, both with positive results, as well as by parasitological culture in NNN medium plus Schneider's medium. For parasitological culture, samples from puncture of bone marrow and lymph node and biopsies of intact skin, skin lesions, lymph node and spleen were examined and resulted in isolation of promastigotes characterized as Leishmania (Leishmania) chagasi by multilocus enzyme electrophoresis. The entomological survey, which was made at peridomicile using CDC light traps, three times a week for three months, did not find sandflies. Additionally, the canine serosurvey conducted in 144 animals around the residence with positive case of LV did not show new cases through rapid immunochromatographic test DPP $®$ and/or through confirmation by ELISA. The results and moving historical of the animal characterize the case as alochthonous, expose the fragility of movement control of animals and highlight the importance of the surveillance for parasitosis in areas unaffected.
\end{abstract}

Keywords: diagnostic, dog, Leishmania.

\footnotetext{
*Recebido em 10 de maio de 2013 e aceito em 20 de junho de 2013.

**Universidade Federal Fluminense, Doutorado em Medicina Veterinária, Niterói, RJ, Brasil e Prefeitura Municipal de Resende, Vigilância em Saúde, Centro de Controle de Zoonoses (CCZ), Resende, RJ, Brasil

***Prefeitura Municipal de Resende, Vigilância em Saúde, CCZ, Resende, RJ, Brasil

****Fundação Oswaldo Cruz (FIOCRUZ), Instituto de Pesquisa Clínica Evandro Chagas (IPEC), Laboratório de Pesquisa Clínica em Dermatozoonoses em Animais Domésticos, Rio de Janeiro, RJ, Brasil

*****FIOCRUZ, IPEC, Laboratório de Vigilância em Leishmanioses, Rio de Janeiro, RJ, Brasil

Autor para correspondência - E-mail: fabiano.figueiredo@ipec.fiocruz.br
} 


\section{Introdução}

A leishmaniose visceral (LV) se tornou um crescente problema de saúde pública no Brasil e em outras áreas do continente americano, sendo uma endemia em franca expansão geográfica (Brasil, 2009). A parasitose, anteriormente considerada uma zoonose rural, hoje afeta centros urbanos de médio e grande porte, em um fenômeno denominado urbanização da LV (Brasil, 2009; 2006; Werneck, 2008). No Brasil, sua forma de transmissão se dá por meio da picada dos vetores Lutzomyia longipalpis e L. cruzi, infectados pelo tripanosomatídeo Leishmania (Leishmania) chagasi (Brasil, 2009; 2006). Na área urbana, o cão (Canis familiaris) é sua principal fonte de infecção (Brasil, 2006).

A primeira epidemia urbana brasileira ocorreu em Teresina, Piauí (Costa et al., 1990), e desde então diversos casos têm sido reportados em outras cidades, como São Luís, no Maranhão (Silva et al., 1997), Belo Horizonte, em Minas Gerais (Luz et al., 2001), Cuiabá, no Mato Grosso (Fontes e Mestre, 2007) e Palmas, no Tocantins (Gloria, 2006). O estado do Rio de Janeiro era considerado indene para LV humana até 1977, quando a doença foi detectada na capital (Marzochi et al., 2009). A $160 \mathrm{~km}$ da mesma encontra-se o município de Resende, localizado na divisa com Minas Gerais, que constitui o terceiro estado em número de casos de LV registrados no Brasil (Mendes et al., 2011).

Na área urbana, o cão (Canis familiaris) é a principal fonte de infecção (Brasil, 2009; 2006)

e a enzootia canina tem precedido a ocorrência de casos humanos (Brasil, 2009; 2006; Silva, 2007; Bevilacqua et al., 2001). Sua importância como reservatório está no contato frequente entre homens e cães e no fato desses animais poderem apresentar infecção assintomática, apesar do alto grau de parasitismo em pele e vísceras (Marzochi et al., 2009).

Este relato apresenta um caso de importação de LV canina para o município de Resende, estado do Rio de Janeiro. A partir de suspeita clínica e prévio exame sorológico positivo, um canino com histórico de residência na cidade de Salinas, MG, porém domiciliado há três anos no município de Resende, RJ, foi submetido à coleta de espécimes clínicos na tentativa de isolamento parasitário e caracterização isoenzimática objetivando comprovar a infecção por Leishmania (L.) chagasi. O município de origem pertence à mesorregião norte de Minas Gerais, sendo essa a de maior incidência da parasitose nesse estado (Mendes et al., 2011).

\section{Relato de caso}

O paciente era um canino, fêmea, adulto, da raça Dachshund, domiciliado no bairro Jardim Brasília, Resende, RJ. O mesmo foi atendido em serviço veterinário particular e após constatação de clínica compatível com LV canina, foram realizados exames sorológicos de Imunofluorescência Indireta e ELISA em rede privada, com obtenção de resultado positivo para diagnóstico de leishmaniose em ambas as avaliações.
O caso foi notificado junto ao Centro de Controle de Zoonoses (CCZ) do município e a equipe do $\mathrm{CCZ}$ procedeu à visita ao domicílio para avaliação clínica do animal e coleta de material biológico para confirmação diagnóstica. Ao exame clínico, o indivíduo apresentava úlcera recoberta por crostas no membro posterior direito, descamação cutânea furfurácea no dorso, caquexia e onicogrifose (Figura 1).

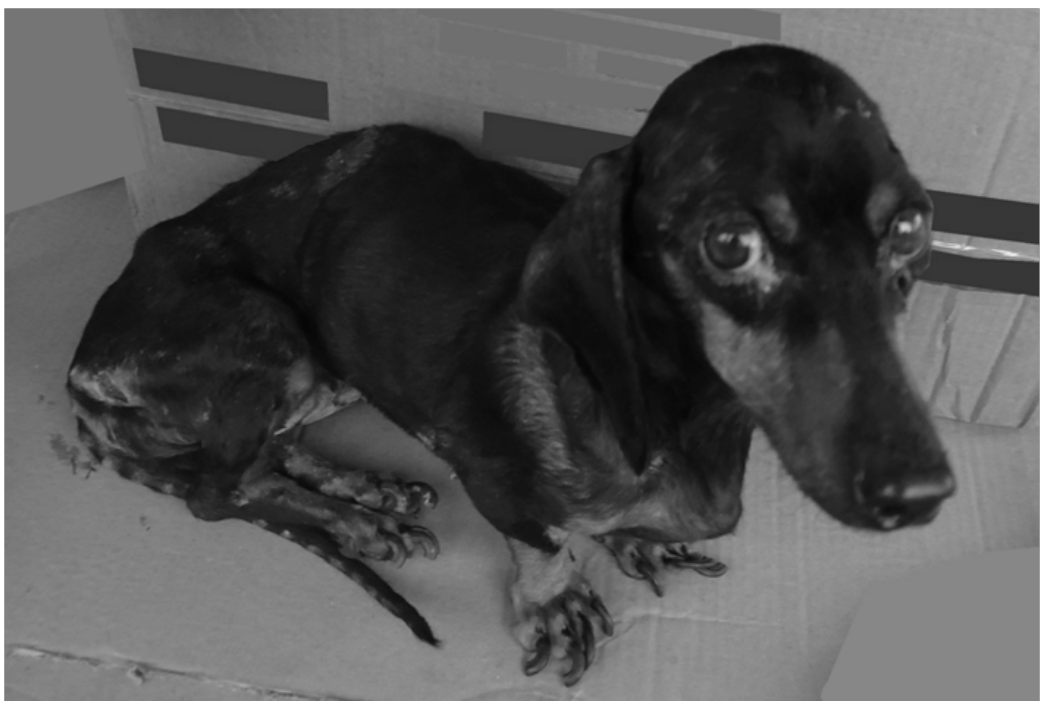

Figura 1: Cão doméstico, raça Dachshund, adulto, infectado por Leishmania (L.) chagasi. Úlcera recoberta por crostas no membro posterior direito, descamação cutânea furfurácea no dorso, caquexia e onicogrifose. Resende, estado do Rio de Janeiro, junho de 2012

Foi realizada coleta de $3 \mathrm{ml}$ de sangue por meio de punção da veia cefálica, sendo esse material encaminhado ao Instituto de Pesquisa Clínica Evandro Chagas (IPEC) da Fundação Oswaldo Cruz (FIOCRUZ), RJ, onde foi realizado teste qualitativo para detecção de anticorpos para Leishmaniose Visceral Canina, o teste rápido imunocromatográfico DPP ${ }^{\circledR}$ (Dual Path Platform) - BioManguinhos ${ }^{\circledR} /$ FIOCRUZ, obtendo resultado positivo, confirmado posteriormente pela realização do teste de ELISA.

Com a ratificação do diagnóstico sorológico, o animal foi encaminhado para o IPEC - FIOCRUZ, onde foi submetido à eutanásia com superdosagem de tiopental sódico $1 \mathrm{~g}$, procedendo-se a necrópsia e coleta de espécimes biológicos, sendo realizadas punções de medula óssea e linfonodos, biópsias de pele íntegra, lesões cutâneas, linfonodo e baço, para tentativa de isolamento parasitário em meio de cultura NNN acrescido de meio Schneider. Em todas as amostras obtevese o isolamento de formas promastigotas compatíveis com Leishmania spp. (Figura 2), exceto nas lesões cutâneas, para as quais houve contaminação da amostra. A amostra isolada foi caracterizada como Leishmania (L.) chagasi, através da eletroforese de isoenzimas (Figura 3). O inquérito entomológico foi realizado utilizando armadilhas luminosas de sucção (modelo CDC), as quais foram introduzidas em ambiente peridomiciliar. As armadilhas foram colocadas às $18 \mathrm{~h}$ e retiradas às $7 \mathrm{~h}$ da manhã seguinte, três vezes por semana, durante três meses. Não foi evidenciada a presença de flebotomíneos, assim como o inquérito sorológico realizado em 144 animais no entorno do domicílio descartou novos casos por meio do teste rápido imunocromatográfico DPP ${ }^{\circledR}$ e/ou confirmação por ELISA. 


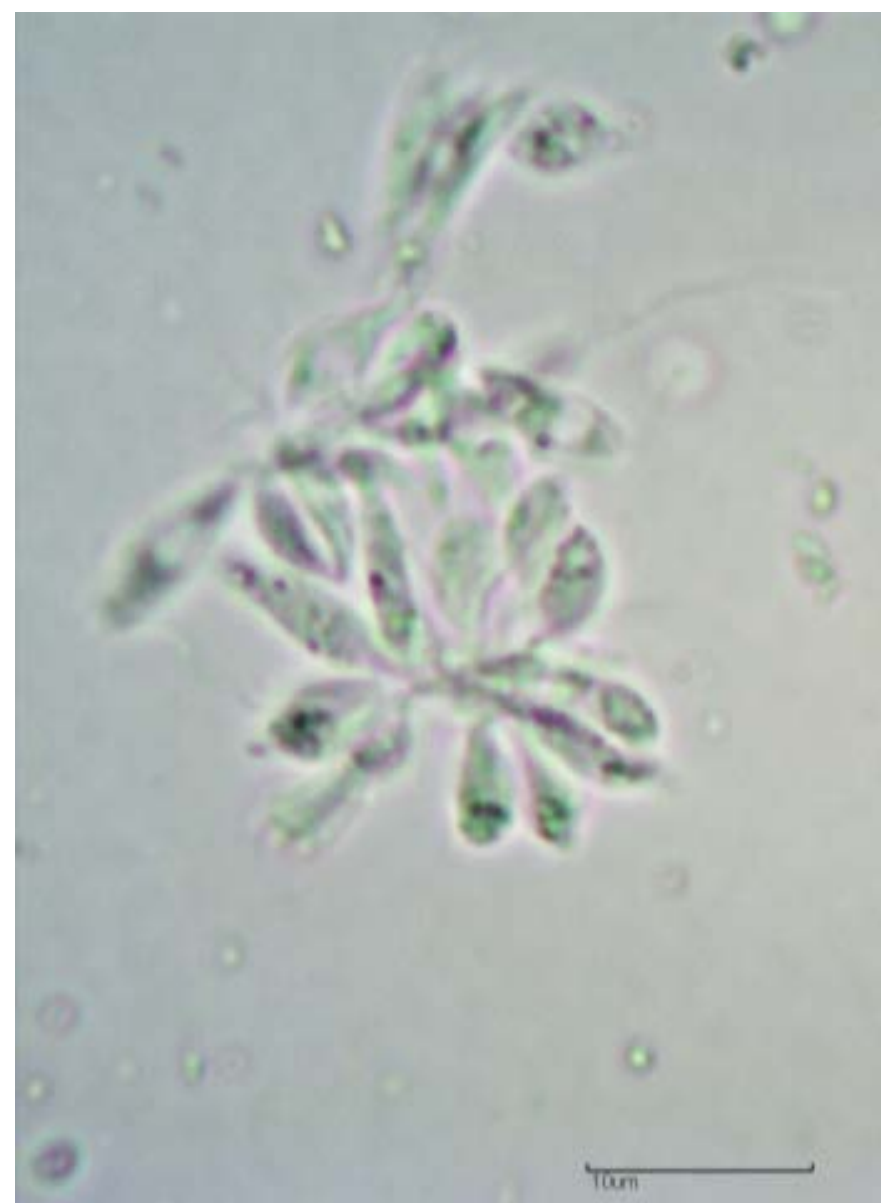

Figura 2: Promastigotas vivas durante exame a fresco da cultura de fragmentos de pele do cão doméstico domiciliado em Resende, estado do Rio de Janeiro, infectado por Leishmania (L.) chagasi. (x1000)

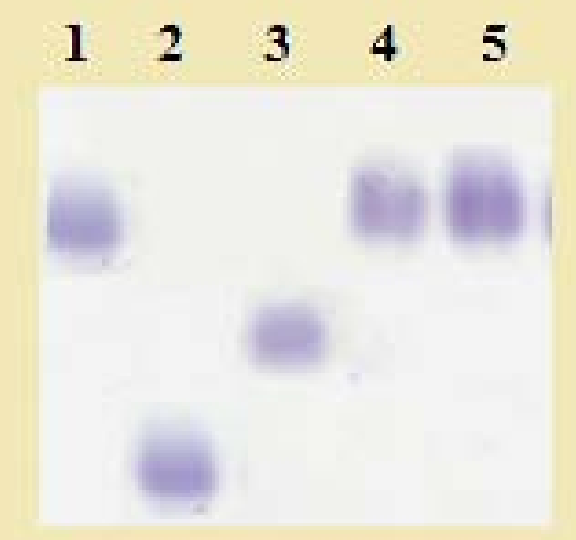

Figura 3: Perfil eletroforético do sistema enzimático fosfoglucose isomerase (GPI). Linhas 1 e 4: amostras de pele do cão doméstico domiciliado em Resende, estado do Rio de Janeiro; linha 2: amostras de referência de Leishmania (L.) amazonensis (IFLA/BR/67/PH8); linha 3: amostra de referência de Leishmania (V.) braziliensis (MHOM/BR/75/M2903) e linha 5: amostra de referência de Leishmania (L.) chagasi (MHOM/BR/74/PP75).

\section{Discussão}

Esse relato evidencia a importação de um caso de LV canina do estado de Minas Gerais para o município de Resende, RJ, onde não havia, até o momento, registro de casos caninos ou humanos da parasitose. De fato, a transferência de animais de áreas endêmicas para áreas não endêmicas coloca a população dessas últimas em risco (Figueiredo et al., 2012) e um caso similar de LV canina, com histórico de deslocamento do animal, foi recentemente descrito em Cachoeira de Macacu, RJ (Silva et al., 2011). A notificação de tais casos salienta a importância das ações de Vigilância para a parasitose.

Resende é um município com grande fluxo populacional, devido à sua localização no eixo Rio de Janeiro - São Paulo, as duas maiores capitais do país, e também devido à presença de grandes indústrias e da Academia Militar das Agulhas Negras, de forma que a cidade recebe pessoas de diversos municípios brasileiros, muitas trazendo consigo seus animais de estimação. Nesse aspecto, a utilização de algumas ferramentas poderia auxiliar na não dispersão da LV canina, tais como: o controle mais rígido do trânsito de cães de áreas endêmicas, implementação do diagnóstico sorológico obrigatório e a notificação compulsória de casos caninos positivos. Nesse sentido, o diagnóstico negativo para o trânsito de cães para áreas indenes deveria ser obrigatório. Além disso, medidas educativas para sensibilizar proprietários de cães e veterinários da rede pública e privada também contribuiriam na tentativa de minimizar o problema (Figueiredo et al., 2012).

Dessa forma, a comunicação entre os serviços públicos e privados torna-se de extrema importância para a notificação e confirmação de casos, sendo que a percepção de veterinários de ambos os setores, público e privado, resulta em benefício para a saúde pública (Bevilacqua et al., 2001), como ocorrido neste relato. A presente notificação do caso permitiu a eliminação de uma fonte de infecção em potencial, por meio da eutanásia do cão positivo, medida recomendada no Brasil (Brasil, 2009; 2006). Os dados apresentados neste estudo demonstram a importância da implantação e implementação da vigilância entomológica e epidemiológica voltadas para LV canina nas áreas indenes, com intuito de detectar a presença do vetor e/ou a enzootia canina antes da instalação do ciclo antropozoonótico, permitindo adotar ações que visem impedir a ocorrência da parasitose em humanos, ou até mesmo preparar os serviços de saúde e a população para o enfrentamento do problema (Figueiredo et al., 2012).

Vale ressaltar que o bairro Jardim Brasília, em Resende, encontra-se em área urbana, onde o cão tem grande importância como reservatório de LV. Dessa forma, o município poderia sofrer um processo similar de urbanização da parasitose como ocorrido em Belo Horizonte, com casos caninos precedendo a infecção humana, caso o ciclo antropozoonótico fosse instalado (Bevilacqua et al., 2001). O problema se agravaria pelo fato de a urbanização ser um fenômeno relativamente novo, pouco se conhecendo sobre a epidemiologia da LV nos focos urbanos, de forma que as relações entre os componentes da cadeia de transmissão nesse cenário parecem ser bem mais complexas e variadas do que no rural (Gontijo e Melo, 2004). 
Um importante passo para a vigilância da LV em Resende, além de estudos relacionados à fauna flebotomínica local e avaliação de cães nas proximidades do caso, seria o estímulo da qualificação de profissionais de saúde, das redes públicas e privadas, para que, em caso de necessidade, o diagnóstico precoce possa ser instituído e as medidas preventivas tomadas em tempo hábil (Figueiredo et al., 2010).

\section{Referências}

BEVILACQUA, P.D.; PAIXÃO, H.H.; MODENA, C.M.; CASTRO, M.C.P.S. Urbanização da leishmaniose visceral em Belo Horizonte. Arquivo Brasileiro de Medicina Veterinária e Zootecnia, v. 53, p. 1-8, 2001.

BRASIL. Guia de vigilância epidemiológica. 7. ed. Brasília, DF: Ministério da Saúde, 2009, 816 p.

BRASIL. Manual de Vigilância e Controle da Leishmaniose Visceral. Brasília, DF: Ministério da Saúde, 2006, 113 p.

COSTA C.H.N.; PEREIRA H.F.; ARAÚJO M.V. Epidemia de leishmaniose visceral no estado do Piauí, Brasil, 1980-1986. Revista Saúde Pública, v. 24, p. 361-372, 1990.

FIGUEIREDO, F.B.; LIMA JUNIOR, F.E.F.J.; TOMIO, E.; INDÁ, F.M.C.; CORREAA, G.L.B.; MADEIRA, M.F. Leishmaniose Visceral Canina: Dois casos autóctones no município de Florianópolis, estado de Santa Catarina. Acta Scientia e Veterinaria , v. 40, n. 1, p. 1-4, 2012.

FIGUEIREDO, F.B.; FILHO, C.J.L.B.; SCHUBACH, E.Y.P.; PEREIRA, S.A.; NASCIMENTO, L.D.E; MADEIRA, M.F. Relato de caso autóctone de leishmaniose visceral canina na zona sul do município do Rio de Janeiro. Revista da Sociedade Brasileira de Medicina Tropical, v. 43, n. 1, p. 98-99, 2010.

GLÓRIA, M.R.B. Leishmaniose Visceral: Situação Epidemiológica e Distribuição Espacial, Município de Palmas, Tocantins. 2006. 86 f. Dissertação (Mestrado) - Curso de Pós-Graduação em Saúde Pública da Fundação Oswaldo Cruz - Escola Nacional de Saúde Pública, Rio de Janeiro, 2006.

GONTIJO, C.M.F.; MELO, M.N. Leishmaniose Visceral no Brasil: quadro atual, desafios e perspectivas. Revista Brasileira de Epidemiologia, v. 7, p. 338-349, 2004.

\section{Conclusões}

Considerando o histórico de deslocamento do animal, este caso foi caracterizado como alóctone.

A importação de um caso de LV canina de uma área endêmica para não endêmica expõe a fragilidade em relação ao controle de trânsito de animais e ressalta a importância das ações de Vigilância para a parasitose em áreas indenes.

LUZ, Z.M.P.; PIMENTA, D.N.; CABRAL, A.L.L.V.; FIÚZA, V.O.P.; RABELLO, A.A. Urbanização das leishmanioses e a baixa resolutividade diagnóstica em municípios da Região Metropolitana de Belo Horizonte. Revista da Sociedade Brasileira de Medicina Tropical, v. 34, p. 249-254, 2001.

MARZOCHI, M.C.A.; FAGUNDES, A.; ANDRADE, M.V.; SOUZA, M.B.; MADEIRA, M.F.; MOUTA-CONFORT, E.; SCHUBACH, A.O.; MARZOCHI, K.B.F. Visceral leishmaniasis in Rio de Janeiro, Brazil: eco-epidemiological aspects and control. Revista da Sociedade Brasileira de Medicina Tropical, v. 42, n. 5, p. 570-580, 2009.

MENDES, C.S.; LOPES, L.S.; TOYOSHIMA, S.H. Determinantes sociais da Leishmaniose Visceral no norte de Minas Gerais. Revista de Economia e Agronegócio, v. 9, n. 1. p.101- 122, 2011.

MESTRE, G.L.C.; FONTES, C.J.F. A expansão da epidemia da leishmaniose visceral no estado de Mato Grosso, 1998-2005. Revista da Sociedade Brasileira de Medicina Tropical, v. 40, n. 1, p. 42-48, 2007.

SILVA, D.A.; PERIÉ, C.S.F.S.; MENDES JÚNIOR, A.A.V.; MADEIRA, M.F.; FIGUEIREDO, F.B. Leishmaniose Visceral Canina em Cachoeira de Macacu, Rio de Janeiro - Relato de Caso. Revista Clínica Veterinária, n. 95, p. 64-68, 2011.

SILVA, F.S. Patologia e patogênese da Leishmaniose Visceral Canina. Revista Tropical - Ciências Agrárias e Biológicas, v.1, n. 1, p. 20-31, 2007.

SILVAA.R.; VIANA G.M.C.; VARONIL C.; PIRES B.; NASCIMENTO M.D.S.D.; COSTA J.M.L. Leishmaniose visceral (Calazar) na ilha de São Luís, Maranhão, Brasil: evolução e perspectivas. Revista da Sociedade Brasileira de Medicina Tropical, v. 30, p. 359-368. 1997.

WERNECK, G.L. Forum: geographic spread and urbanization of visceral leishmaniasis in Brazil. Introduction. Caderno de Saúde Pública, v. 24, n. 12, p. 2937-2940, 2008. 